\title{
DETECTING INFORMAL SETTLEMENTS FROM QUICKBIRD DATA IN RIO DE JANEIRO USING AN OBJECT BASED APPROACH
}

\author{
P. Hofmann ${ }^{c}$, Josef Strobl ${ }^{a}$, Thomas Blaschke ${ }^{a}$, Hermann Kux ${ }^{b}$
a Z_GIS, Zentrum für Geoinformatik, Paris-Lodron-Universität Salzburg, Hellbrunnerstraße 34, A-5020 Salzburg - (josef.strobl,thomas.blaschke)@sbg.ac.at
${ }^{\mathrm{b}}$ INPE, São José dos Campos - SP, Av. dos Astronautas, 1758 - Jardim da Granja, CEP 12227-010 -
hermann@dsr.inpe.br
c Paris-Lodron-Universität Salzburg, Hellbrunnerstraße 34, A-5020 Salzburg - hofmann@ipi.uni-hannover.de

KEY WORDS: Informal settlements, urban remote sensing, object based image analysis, fuzzy logic

\begin{abstract}
:
Informal settlements behave very dynamical over space and time and the number of people living in such housing areas is growing worldwide. The reasons for this dynamical behaviour are manifold and are not matter of this article. Nevertheless, informal settlements represent a status quo of housing and living conditions which is from a humanitarian point of view in the most cases below acceptable levels. Sub-standard sanitary situations and high crime rates are only a few of attributes which go aside with the phenomenon informal settlement. Due to their informal character, reliable and accurate data about informal settlements and their inhabitants is rarely available. On the other side there is a strong need to transform informal into formal settlements and to gain more control about the actual spatial development of informal settlements. Consequently, reliable procedures for detecting and monitoring the spatial behaviour of informal settlements are required in order to react at an early stage to changing housing situations. Thus, obtaining spatial information about informal settlement areas which is up to date is vital for any actions of enhancement in terms of urban or regional planning. For these tasks, conventional data sources, such as maps, statistics or even GIS data are usually obsolete, not available, not as accurate as needed or do not hold the information needed. The article present will demonstrate how informal settlements can be detected from VHR satellite image data using an object based approach of image analysis. The classified areas can act as basic information for further GIS-based tasks.
\end{abstract}

\section{INTRODUCTION}

\subsection{Informal settlements}

Informal settlements are usually a phenomenon which mostly occurs in developing and newly industrializing countries. Although different definitions of informal settlement do exist, slum, favella, squatter settlement or shanty town are commonly used synonyms for this special type of settlement. Nevertheless, the UN (UNSTAT, 2005) define informal settlements as:

„1. areas where groups of housing units have been constructed on land that the occupants have no legal claim to, or occupy illegally; 2. unplanned settlements and areas where housing is not in compliance with current planning and building regulations (unauthorized housing)."

Both definitions are obviously emphasising the illegal character of informal settlements. In contrast, the definition of Mason, O.S. \& Fraser, C., 1998 takes the environmental, socioeconomic and living conditions more into account. They describe informal settlements as:

“... dense settlements comprising communities housed in self-constructed shelters under conditions of informal or traditional land tenure ... . They are a common feature of developing countries and are typically the product of an urgent need for shelter by the urban poor. As such they are characterised by a dense proliferation of small, makeshift shelters built from diverse materials (such as plastic, tin sheeting and wooden planks), by degradation of the local ecosystem (for example, erosion and poor water quality and sanitation) and by severe social problems."

Independent of these different definitions, according to UNHABITAT (UN-HABITAT, 2006a) the number of people living in slums, favellas or shanty towns worldwide will grow from approx. 1.0 Billion in 2005 to 1.2 Billion in 2010 and 1.5 Billion in 2020. Thus, from the perspective of an urban or regional planner, as well as from the perspective of local or regional authorities, informal settlements might become a more and more challenging problem in the years to come. Programs, such as the Global Campaign For Secure Tenure (UNHABITAT, 2006) are emphasising this challenging character.

\subsection{Monitoring and detecting informal settlements}

From a methodological point of view, the challenge lies in having appropriate methods to detect and monitor the spatial behaviour of informal settlements reliably (see Lemma, T. et al., 2005, Mason, S.O. et al., 1998, Dare, P.M. \& Fraser, C.S., 2001, Kuffer, M., 2003 and Radnaabazar, G. et al., 2004). Regarding available data sources, remotely sensed imagery from satellites therefore offers a well suited data source. To benefit from the advantages of this data source and to obtain the information as needed, adequate methods for analysing remote sensing data are necessary. In an ideal case, these methods can be applied without the need of expert knowledge and human interaction. In practise easiness of use and the degree of automation for information extraction from imagery depends on the data used and the phenomena to be extracted from the 
image. In this context, informal settlements show a relatively high inner-structural heterogeneity which leads to particularly hard describable patterns in the image. This hampers the generation of an automated detection process which is easy to use. Nevertheless, in Hofmann, P., 2001 first results of detecting informal settlements from IKONOS data in Cape Town showed the principle feasibilities by using an objectoriented approach. The results were promising but seamed to be very dependent on the data used. Especially the relative complex class-hierarchy turned out not to be flexible enough for being applied successfully to other scenes. Applying the developed extraction methods from Hofmann, P., 2001 to the QuickBird-Scene used in this paper showed that several adoptions were necessary. Besides, we applied some fundamental changes in the methodology: while the development of the rule-base in Hofmann, P., 2001 was mainly driven by the data used, the rule-base developed here took more the phenomena and their ontologies into account. Thus, the number of classes and sub-classes was noticeably reduced and the use of class descriptions by nearest neighbour was completely renounced, which led to classes only described by fuzzy membership functions and their combinations. Nevertheless, in both cases the basic strategy and the initial segmentation were similar: starting with eCognition's multi resolution segmentation as the initial segmentation a classification of the generated image objects follows and ends in an iterative process of a knowledge-based object enhancement and (re-) classification (see Figure 1).
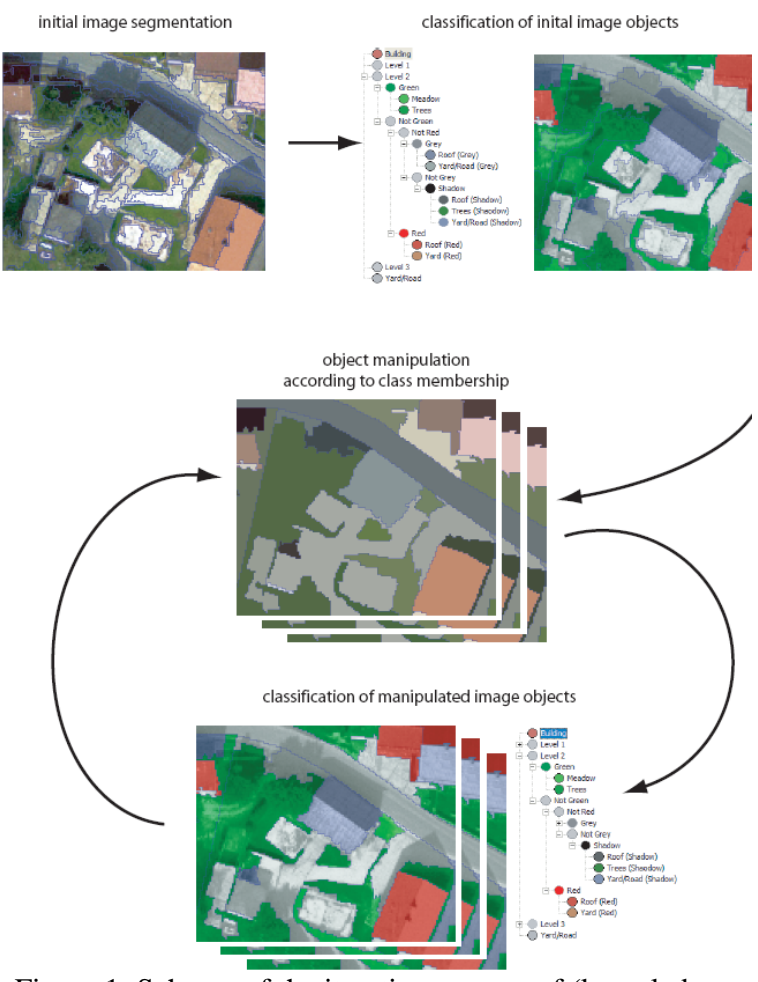

Figure 1: Schema of the iterative process of (knowledgebased) image segmentation and classification.

\section{THE ONTOLOGY OF INFORMAL SETTLEMENTS}

\subsection{What are informal settlements ?}

Besides the two basic definitions already given in the first chapter, to describe what an informal settlement is depends strongly on the context respectively the point of view. Without going deep into details about theoretical aspects of ontologies, describing "what is an informal settlement" is a special case of describing phenomena of the (real) world. In general, this is done by describing knowledge about the phenomenon from a certain point of view using a certain language. Thus, the ontological description of "informal settlement" can be understood as the representation of knowledge about "informal settlement" from a certain point of view using a defined language. The language has to follow certain rules which are described in Guarino, N., 1998 in more detail. Nevertheless, it is obvious that as many knowledge representations of a phenomenon can exist as points of view do (see Fonseca, T.F., 2001). These points of view are commonly named domains. As such, in the context of image analysis, we can identify the two basic domains: image domain and real world domain. Both domains are interacting with each other as Figure 2 illustrates.

\subsection{Informal settlements from the perspective of different domains}

The description from the point of view of the real world domain in this context describes a phenomenon by its general observable properties, i.e. what is typical or even unique for the phenomenon (here: informal settlements) in general. From the point of view of the image domain, these properties have to be measurable (i.e. detectable) in the image. These properties are commonly understood as the signature or pattern of a phenomenon independent of possible methods of image processing to quantify them.

Using a taxonomical description in terms of "informal settlement is a special case of settlement" helps to identify unique properties of informal settlements and common properties with other types of settlement. Describing the spatial relationships between objects of different classes goes beyond the description of patterns but is nevertheless part of the phenomena's ontologies. I.e. these are descriptions of the phenomena from the real-world domain's point of view. Within the context of informal settlements, such relationships have to take into account the dense structure of housing and infrastructure, respectively the informal character of these subelements. When referencing objects of such classes, especially when describing a spatial context relation, of course their ontologies have to be described as well, in order to detect them in the image appropriately. Analogous to the description of settlement areas and informal settlements it has to be described what is typical for an informal house, informal infrastructure or vegetation in the real world domain and in the image domain (see Figure 3).

A further issue typical for settlement structures within urban areas is their segregation. While the reasons for segregation of city dwellers are usually more socio-economic, the phenomenon itself can be observed by locally concentrated patterns of different settlement structures. Vice versa, in some cases we can describe knowledge about the exclusion of certain types of settlement within other settlement areas. In the context of detecting informal settlements we can exclude a priori the occurrence of formal housing structures within informal settlement areas. 


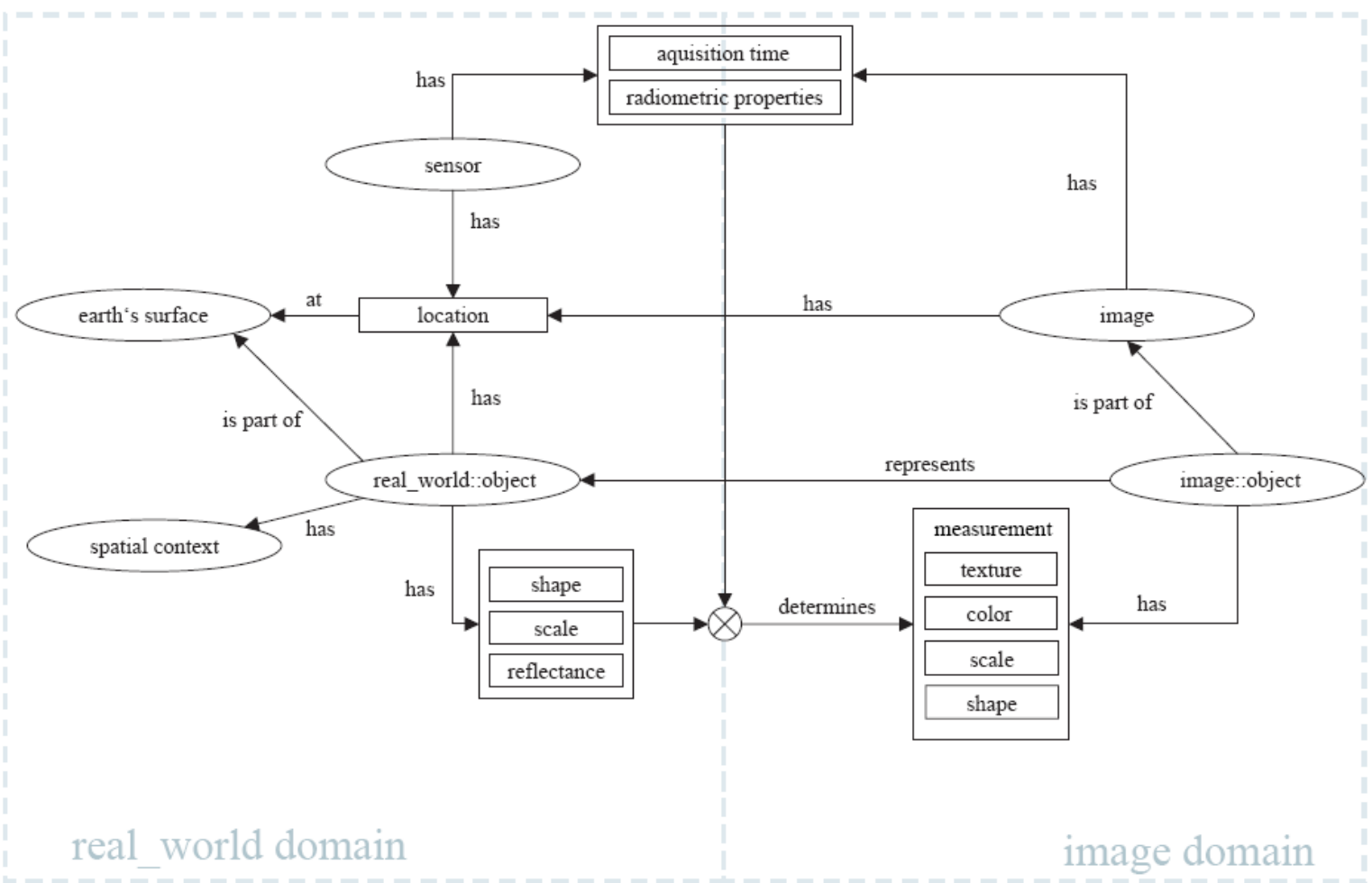

Figure 2. Relationships between objects of the real world domain and the image domain.

\section{IMAGE SEGMENTATION}

\subsection{Segmentation strategy based upon the ontology}

Regarding the ontology of informal settlements, especially the spatial relationships between (informal) settlement and its subobjects buildings, road network and vegetation (Figure 3), it is obvious that the image segmentation has to generate image objects representing settlement areas and the named subobjects. Using eCognition with its multi-resolution segmentation method for this purpose, means generating two segmentation levels, wherein the top level more or less represents settlement areas and other objects of comparable size, while the base level holds image objects which coincide with small houses, small road segments and small vegetation areas. The image objects of both segmentation levels are linked to each other in terms of a hierarchical net of objects which is depicted by a tree-structure (see Hofmann, P., 2001, Baatz, M. et al., 2004, Benz et al., 2004). This makes it possible in principle to describe later on spatial relationships between settlement areas and small houses, small road segments and small vegetation areas as well as the described neighbourhood relations reflecting aspects of segregation.

Regarding the segmentation parameters, according to Baatz, $\mathrm{M}$. et al., 2004 the image objects created by the initial segmentation should best suit the image analysis purposes. I.e. the image segmentation should lead to image objects which best suit the ontologies of the desired classes. In practise this leads to many trial and error tasks in order to find the best suited segmentation parameters for the initial segmentation. Thereby, usually not all desired objects will be outlined semantically perfect, i.e. some objects are over-segmented, while others are under-segmented. Thus, the optimum segmentation parameters are those which obviously generate the least over- or undersegmentations. In the scenes used here and in Hofmann, P., 2001 colour contrast in informal settlement areas is relatively

\begin{tabular}{|c|c|c|c|}
\hline \multicolumn{2}{|c|}{ Scene } & $\begin{array}{c}\text { IKONOS Cape } \\
\text { Town }\end{array}$ & $\begin{array}{l}\text { QuickBird Rio } \\
\text { de Janeiro }\end{array}$ \\
\hline \multicolumn{2}{|c|}{ Location } & $\begin{array}{c}\text { Cape Town } \\
\text { Nyanga/Crossr } \\
\text { oads) }\end{array}$ & $\begin{array}{c}\text { Rio de Janeiro } \\
\text { (Ilha do } \\
\text { Governador) }\end{array}$ \\
\hline \multicolumn{4}{|c|}{ Spatial resolution } \\
\hline \multirow{5}{*}{ Channel } & pan & $1.0 \mathrm{~m}$ & - \\
\hline & blue & $4.0 \mathrm{~m}$ & $0.69 \mathrm{~m}$ \\
\hline & green & $4.0 \mathrm{~m}$ & $0.69 \mathrm{~m}$ \\
\hline & red & $4.0 \mathrm{~m}$ & $0.69 \mathrm{~m}$ \\
\hline & nir & $4.0 \mathrm{~m}$ & $0.69 \mathrm{~m}$ \\
\hline \multicolumn{4}{|c|}{ Radiometric resolution } \\
\hline \multirow{5}{*}{ Channel } & pan & 11bit (16bit) & 11bit (16bit) \\
\hline & blue & 11bit (16bit) & 11 bit (16bit) \\
\hline & green & 11bit (16bit) & 11bit (16bit) \\
\hline & red & 11bit (16bit) & 11 bit (16bit) \\
\hline & nir & 11 bit (16bit) & 11 bit (16bit) \\
\hline \multicolumn{4}{|c|}{ Band widths } \\
\hline \multirow{5}{*}{ Channel } & pan & $450-900 \mathrm{~nm}$ & $450-900 \mathrm{~nm}$ \\
\hline & blue & $450-520 \mathrm{~nm}$ & $450-520 \mathrm{~nm}$ \\
\hline & green & $510-600 \mathrm{~nm}$ & $520-600 \mathrm{~nm}$ \\
\hline & red & $630-700 \mathrm{~nm}$ & $630-690 \mathrm{~nm}$ \\
\hline & nir & $760-850 \mathrm{~nm}$ & $760-900 \mathrm{~nm}$ \\
\hline \multicolumn{2}{|c|}{ pre-processing by provider } & $\begin{array}{c}\text { reclassification } \\
\text { to } 11 \text { classes }\end{array}$ & pan-sharpening \\
\hline \multicolumn{2}{|c|}{ pre-processing by customer } & pan-sharpening & $\begin{array}{c}\text { reclassification } \\
\text { to } 11 \text { classes }\end{array}$ \\
\hline
\end{tabular}

Table 1: Properties of the image data used in Hofmann, P., 2001 and in the research work described here.

low. Thus, the segmentation of small houses respectively single shacks succeeded in both scenes only partially. However, Hofmann, P., 2001 has already demonstrated that it is not necessary to identify each individual house to classify different 
types of settlement areas. Moreover, it turned out that most of the houses' shadows as well as roofs with higher contrast to their environment (mostly houses with red roofs) could be segmented well enough in order to identify different housing structures by the generated segments. These different housing structures were used later on successfully to differentiate informal settlement areas from other areas. For the ontology of informal settlements, from the point of view of the image domain, the spatial relationship between (informal) settlement and (small) houses must be expressed indirectly by these detectable indicators (i.e. roofs and shadows) and their respective properties (Figure 3 ).

\subsection{Generating an adequate knowledge description}

Although the complexities of the class-hierarchies are very different, the base strategy of beginning with so-called "level super-classes" applies to both hierarchies. With these superclasses objects belonging to the base- or top-segmentation level are separated semantically by using a crisp membership function describing the feature "Level". Following the spatial relationships of sub- and super-objects in the ontology of informal settlements, the classes settlement area, formal settlement and informal settlement are sub-classes of top level, whereas formal settlement and informal settlement are subclasses of settlement. This way, properties which are typical for settlements in general are described in the class settlement and

\subsection{Segmentation strategy based upon image data}

Although the segmentation parameters used in Hofmann, P., 2001 led to well usable image objects with the above described restrictions and although there is only little difference between the image properties of the scenes (see Table 1), using identical parameters for segmenting the QuickBird-scene did not lead to as useful image objects as in the IKONOS scene. In fact the average object size in the IKONOS scene was in the top level at approx. $3,165 \mathrm{~m}^{2}$ and in the base level at approx. $49 \mathrm{~m}^{2}$. Within informal settlements the average object size was at $14,608 \mathrm{~m}^{2}$ and $34 \mathrm{~m}^{2}$ respectively. In the QuickBird scene using identical segmentation parameters the average object size in the top level was at $1,045 \mathrm{~m}^{2}$ and at $25 \mathrm{~m}^{2}$ in the base level. Within informal settlements the respective average object sizes were $1,273 \mathrm{~m}^{2}$ and $15 \mathrm{~m}^{2}$. Regarding the objects' properties, only slight differences of the spectral properties were observable, but properties describing the objects' shape and structure were varying. While it was not quite clear which differences in the image properties finally led to these differences in the object properties, we assumed that one key feature is the higher spatial resolution of the QuickBird scene. Hence, we decided to multiply the 'scale parameter' according to the ratio of the resolutions of both sensors and to segment the QuickBird-scene with 'scale parameters' of 144 instead of 100 (top-level) and 14 instead of 10 (base-level). Although this approach follows more heuristic assumptions than provable calculations the resulting objects now more met the ontological strings and were visually more comparable.

\section{FROM ONTOLOGIES TO A RULE BASE}

While in Hofmann, P., 2001 the design of the knowledge-base was more or less orienting on the data used, which led to a relatively complex class hierarchy with likewise complex class descriptions, the rule base developed here is more orienting to the phenomena and their ontologies. Thus, the class-hierarchy generated here is more pruned, focusing on relevant properties according to the ontology and consequently more transparent. Having in mind to apply the class-hierarchy to several comparable scenes, the improved transparency simplifies necessary incurring and maintenance tasks. properties are common to both types of settlement. This hierarchical structure reflects the "is a" relations of the ontologies described in Figure 3. The classes red roofs, small shadows/dark objects, bright small roofs/objects and vegetation are sub-classes of base level. Simultaneously, the classes of the base level are acting as indicators for (informal) settlement as described in Figure 3, i.e. the "has" relations.

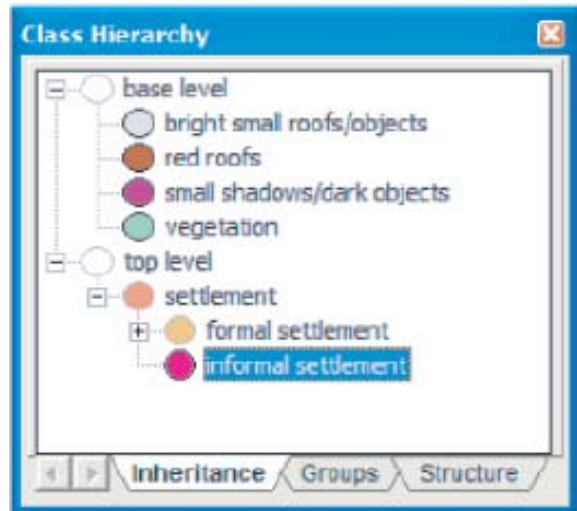

Figure 4: Class hierarchy of settlement, formal and informal settlement. 
Since it was not possible to segment and identify single houses with informal character the density of the indicators red roofs, small shadows/dark objects, bright small roofs/objects was used to identify settlements and informal settlements. This was done by using and combining appropriate membership functions for the features "Asymmetry" and "Area of Sub-Objects" with the features "Rel. area of bright small roofs/objects", "Rel. area of small shadows/dark objects" and "Rel. area of red roofs". The feature "Avrg. mean diff to neighbors of sub-objects" in the nirchannel was used to describe the relatively high spectral heterogeneity within settlement areas. "Asymmetry" was used to differentiate settlement areas from other elongated objects like rail roads or roads. Informal settlements then could be differentiated from other types of settlement by a smaller "Area of Sub-Objects", an explicit lower "Rel. area of red roofs" combined with an explicit lower "Rel. area of bright small roofs/objects", but an explicit higher "Rel. area of small shadows/dark objects". The informal character of the road network within informal settlements was expresses by a relative low value for "Asymmetry of sub-objects: mean". Since a higher value for this feature indicates more elongated objects present (e.g. regular road segments), a lower value indicates the opposite (Figure 5).

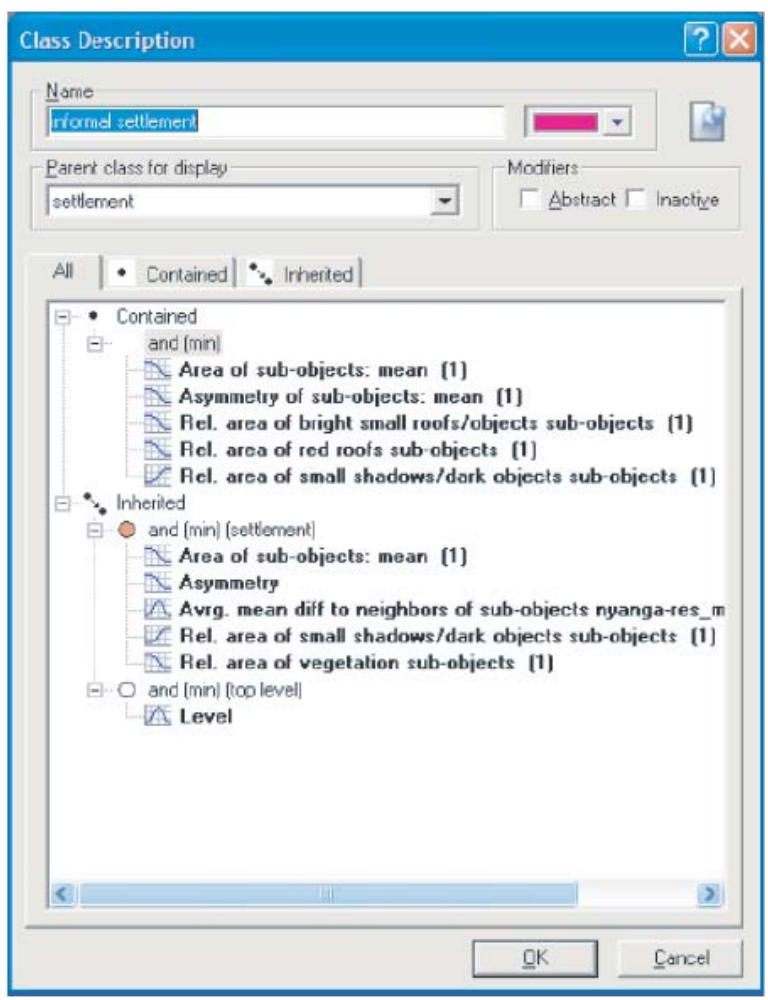

Figure 5: Class description of informal settlement by appropriate fuzzy-membership functions.

The class formal settlement finally is simply described by the fuzzy-logical negation (inversion) of informal settlement combined with a fuzzy-limiting value for "Area" of more than $1800-1900 \mathrm{~m}^{2}$. Since formal settlement is a sub-class of settlement, it inherits all its feature descriptions and is simultaneously the 'opposite' of informal settlement, i.e. all settlement areas which are not informal.

\subsection{Knowledge based iterative segmentation}

The class descriptions presented until now were mainly focusing on describing aspects of color, shape, texture and structure, i.e. the physical properties of (informal) settlements and their structures. As was shown already in this article, one typical property of settlement areas - especially in urban areas - is to be segregated according to different features which are not all directly detectable from remote sensing data. When regarding spatial neighbourhood relationships between different types of housing, it is very unlikely that formal settlement areas are completely surrounded by structures of informal settlements. Notably the inverse constellation is very likely so that preferably within transition zones from formal to informal settlements such mixed areas do occur. However, applying the class-hierarchy described before leads to a the classification result with some misclassified formal settlement objects which are either very close to informal settlement objects or even embedded by such areas. Although the objects fulfil the criteria of settlement, they do not for some reason for informal settlement and are thus classified as formal settlement (the inverse of informal settlement). These circumstances seam to infringe the 'rules' of segregation, i.e. it seams to be very likely, that these objects are truly misclassified and should be assigned to informal settlement. In order to identify such formal settlement objects which are embedded by informal settlement objects, we used the feature "Rel. area of [class] neighbourobjects", whereas informal settlement was used for [class]. The distance was set to 0.0 , which means we are looking for objects at direct neighbourhood. In order to obtain more realistic values for measuring embedding, we fused all neighbouring objects classified as informal settlement by the so-called 'classification based segmentation'. This way, the higher the "Rel. area of informal settlement neighbour-objects" is, the more it is embedded. To realise the measurement after the object fusion, a new class was created as a sub-class of formal settlement, but with the additional property "Rel. area of informal settlement neighbour-objects 0.0 ” fuzzy-more than $0.3-0.5$. This means, all objects classified as formal settlement and whose share of area of informal settlement in the direct neighbourhood is fuzzy-more than $30 \%-50 \%$ are assigned to the new class formal surrounded by informal (Figure 6).

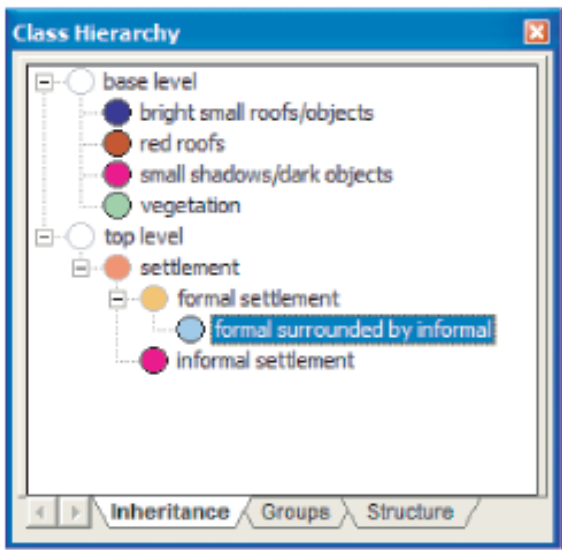

Figure 6: Extended class-hierarchy for detecting embedded formal settlement areas.

Since we stated before that these objects are actually misclassified - i.e. they should be classified as informal settlement instead of formal settlement - the class formal surrounded by informal was assigned to the semantic group (see Baatz, M. et al., 2004) informal settlement. This semantic assignment in consequence raises the number of informal settlement objects and of course the global area of this class. For the formal settlement neighbours of the prior formal surrounded by informal neighbours this means: they now have a direct neighbourhood to informal settlement. If their value for 
"Rel. area of informal settlement neighbour-objects 0.0 " is fuzzy-more than $0.3-0.5$ they are now embedded by informal settlement too. Fusing all neighbouring informal settlement objects leads for all objects embedded by informal settlement to a higher value of "Rel. area of informal settlement neighbourobjects 0.0 ". Applying a reclassification after the fusion leads to the initial situation but with raised informal settlement areas.

Repeating this sequence of (re-)classification and fusion leads to an iterative process that will stop as soon as there are no further formal settlement objects embedded by informal settlement present, or the process is aborted by the operator. Saving the sequence of (re-)classification and fusion in a socalled 'protocol' leads to a program-like knowledge-based region-growing procedure. In this form it is possible to re-apply the procedure or modify it wherever necessary.

\section{ACCURACY ASSESSMENT}

To assess the accuracy of the classification process in terms of analysing the error or confusion matrix, we used the results of a manual classification as reference areas. The overall accuracy for the classification before applying the iterative approach was at $47 \%$. After applying the iterative process the overall accuracy could be increased to $68 \%$. However, when extracting objects of only one class, calculating the errors of commission and omission (users and producer accuracy) actually leads to a misinterpretation of the error matrix, since there is usually no reference for the non-desired classes available (here: formal settlement). I.e. the overall accuracy only measures the matching of reference pixel and classified pixel inside the reference areas but does not care about the false positives outside the reference areas and the false negatives (not classified pixel) inside the reference areas. Regarding the measures 'Best Classification Result' and 'Classification Stability' offered by eCognition (see Baatz, M. et al., 2004, pp. 160 - 163), there are slight enhancements observable, too when applying the iterative process: The mean values for 'Best Classification Result' and 'Classification Stability' within the reference areas could be raised from 0.92 to 0.94 after applying the iterative process.

\section{CONCLUSION AND OUTLOOK}

In the paper present we have demonstrated how informal settlements can be extracted from VHR satellite imagery using an object based approach. In contrast to former approaches such as in Hofmann, P., 2001 the image segmentation and knowledge description was more driven by the ontologies of the desired objects than by the data used. Additionally, in comparison to Hofmann, P., 2001 we generated and applied a simplified but more precise class-hierarchy, which finaly led to a more transperent and easier maintainable extraction process. Allthough some data related adaptations of the segmentation parameters were necessary, by formulating and applying imageindependent knowledge about the spatial behaviour of the desired objects (here: segregation) led to an iterative process which produced enhanced objects and more accurate classification results.

\section{REFERENCES}

Benz, U. et al., 2004: Multi-resolution, object-oriented fuzzy analysis of remote sensing data for GIS-ready information. In: ISPRS Journal of Photogrammetry \& Remote Sensing 58 (2004), pp. 239-258.
Dare, P.M. \& Fraser, C.S., 2001: Mapping informal settlements using high resolution satellite imagery. In: Int. J. of Remote Sensomg, Vol. 22, No. 8 pp. 1399-1301.

Baatz, M. et al., 2004: eCognition 4 User Guide, $4^{\text {th }}$ Ed., Munich, 2004.

Fonseca, T.F., 2001: Ontology-driven Geographic Information Systems, University of Maine, Diss., 2001.

Guarino, N., 1998: Formal Ontology and Information System. In: Proceedings of the International Conference on Formal Ontology in Information Systems, IOS Press, Amsterdam, 1998, pp. 3-15.

Hofmann, P., 2001: Detecting Informal Settlements from IKONOS Image Data Using Methods of Object Oriented Image Analysis - An Example from Cape Town (South Africa). In: Jürgens, C. (Ed.): Remote Sensing of Urban Areas / Fernerkundung in urbanen Räumen. Regensburg: University Regensburg, Germany, 2001, pp. 41-42.

Kuffer, M., 2003: Monitoring the Dynamics of Informal Settlements in Dar Es Salaam by Remote Sensing: Exploring the Use of SPOT, ERS and Small Format Aerial Photography. In: Schrenk, M. (Ed.): Proceedings CORP 2003, pp. 473-483.

Lemma, T. et al., 2005: A Participatory Approach to Monitoring Slum Conditions.

http://www.itc.nl/library/papers_2005/conf/sliuzas_par.pdf (accessed 2006).

Mason, O.S. \& Fraser, C., 1998: Image sources for informal settlement management. In: Photogrammetric Record, Vol. 92 (1998), No. 16, pp. 313-330.

Mason, S.O. et al., 1998: Spatial Decision Support Systems for the Management of Informal Settlements. In: Comput., Environ. and Urban Systems, Vol. 21, No 3/4, pp. 189-208.

Radnaabazar, G. et al., 2004: Monitoring the development of informal settlements in Ulanbaatar, Mongolia.

http://www.schrenk.at/CORP_CD_2004/archiv/papers/CORP20 04_RADNAABAZAR_KUFFER_HOFSTEE.PDF (accessed 2006).

UN-HABITAT, 2006:

http://www.unhabitat.org/campaigns/tenure/cws.asp (accessed 2006).

UN-HABITAT, 2006a :

http://www.unchs.org/programmes/guo/statistics.asp (accessed 2006).

UNSTAT, 2005:

http://unstats.un.org/unsd/environmentgl/gesform.asp?getitem= 665 (accessed 2006). 